
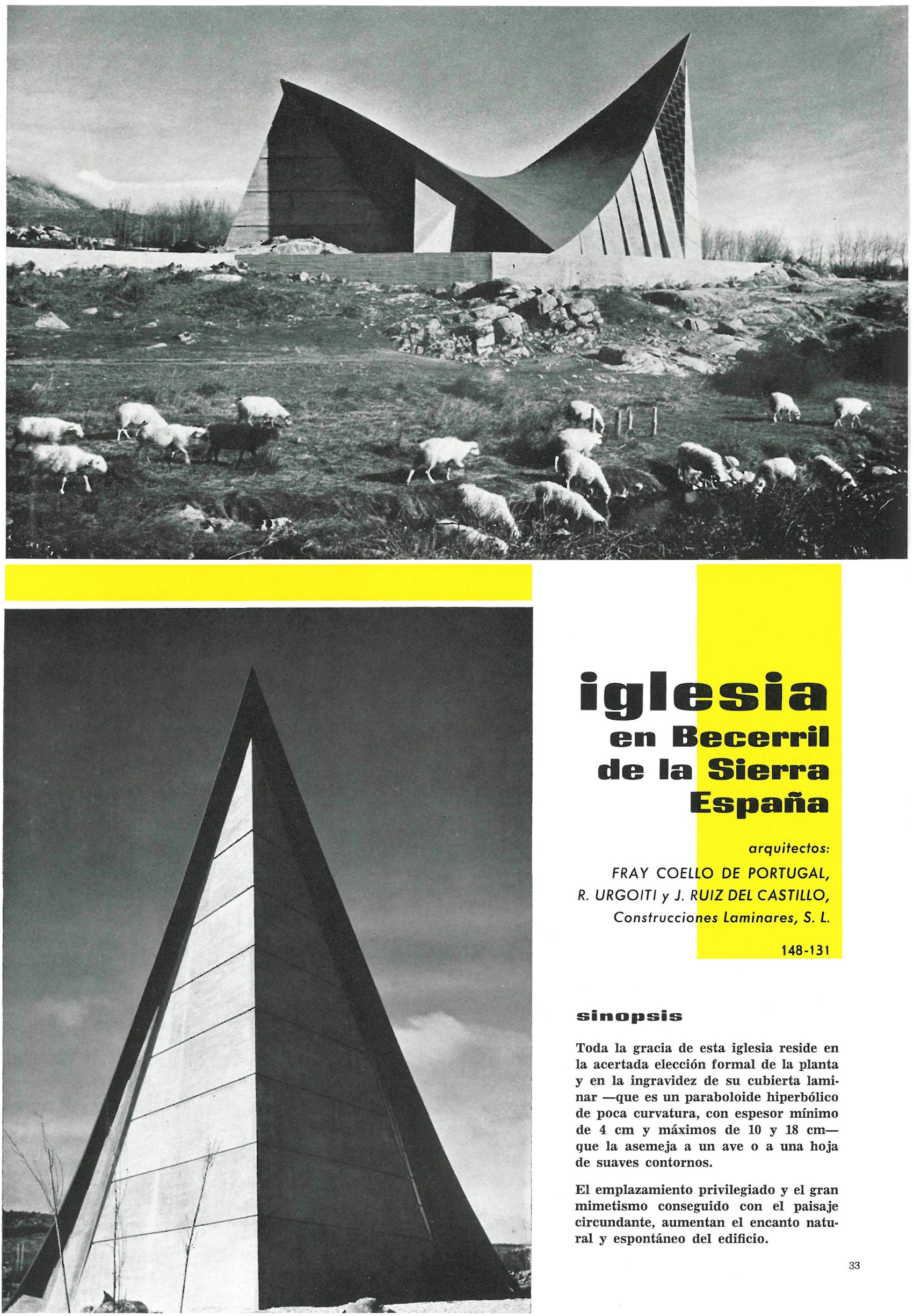

\section{iglesia en Becerril de la Siemra España}

arquitectos:

FRAY COELLO DE PORTUGAL, R. URGOITI Y J. RUIZ DEL CASTILLO,

Construcciones Laminares, $S$. $L$.

$148-13$

\section{sinopsis}

Toda la gracia de esta iglesia reside en la acertada elección formal de la planta y en la ingravidez de su cubierta laminar -que es un paraboloide hiperbólico de poca curvatura, con espesor mínimo de $4 \mathrm{~cm}$ y máximos de 10 y $18 \mathrm{~cm}-$ que la asemeja a un ave o a una hoja de suaves contornos.

El emplazamiento privilegiado y el gran mimetismo conseguido con el paisaje circundante, aumentan el encanto natural y espontáneo del edificio. 


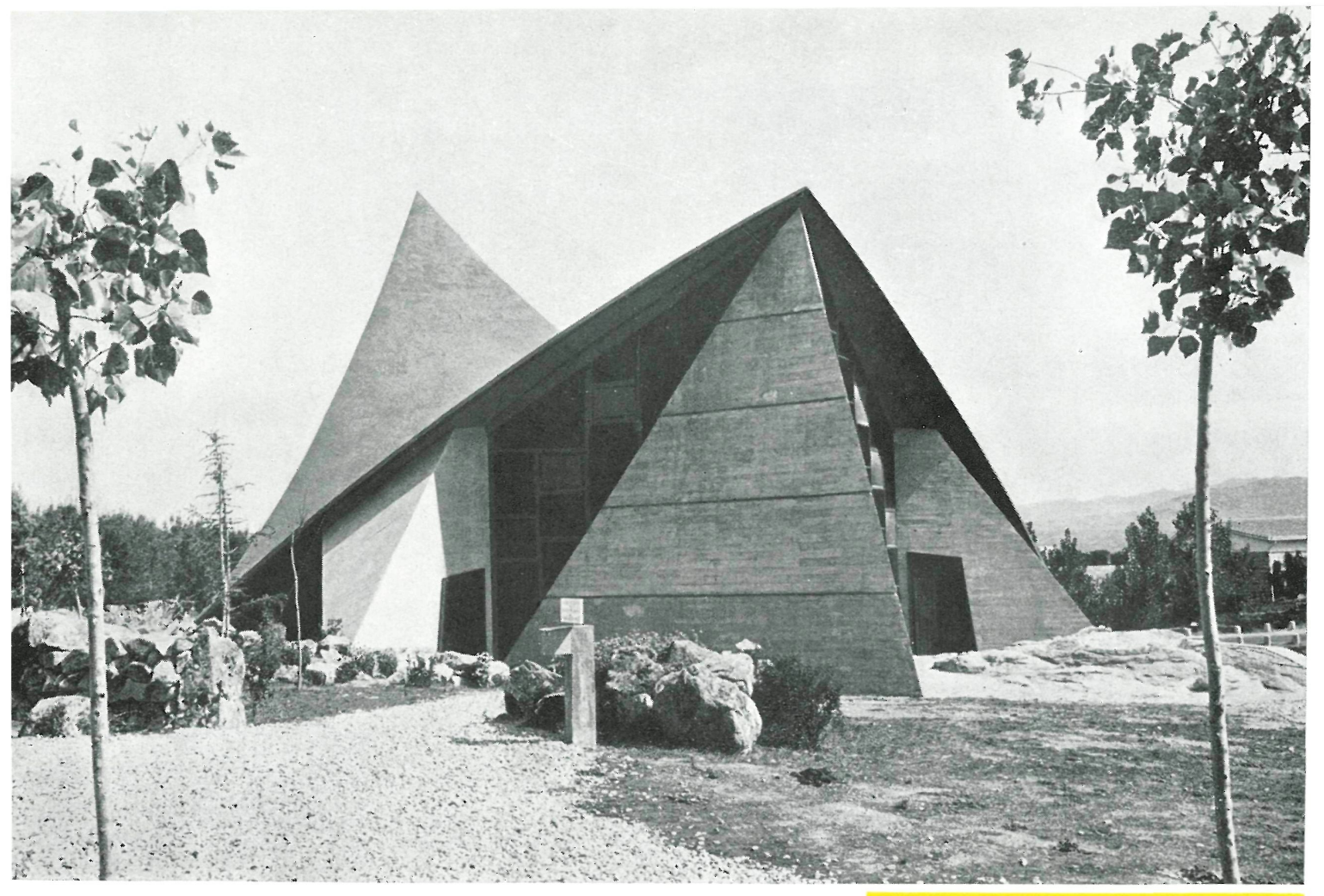

El templo ha sido proyectado en un asentamiento privilegiado, en un triángulo de campo agreste bordeado por carretera, el río y el puente, con largas vistas en todas direcciones y terreno movido de musgo y peñas desperdigadas, con el fondo montañoso turbulento de La Maliciosa.

El diseño, sin desvirtuar su legitimidad estructural, se supeditó a un prurito morfológico condicionado por el lugar. La cresta ágil de la silueta montañosa reclamaba el contra-
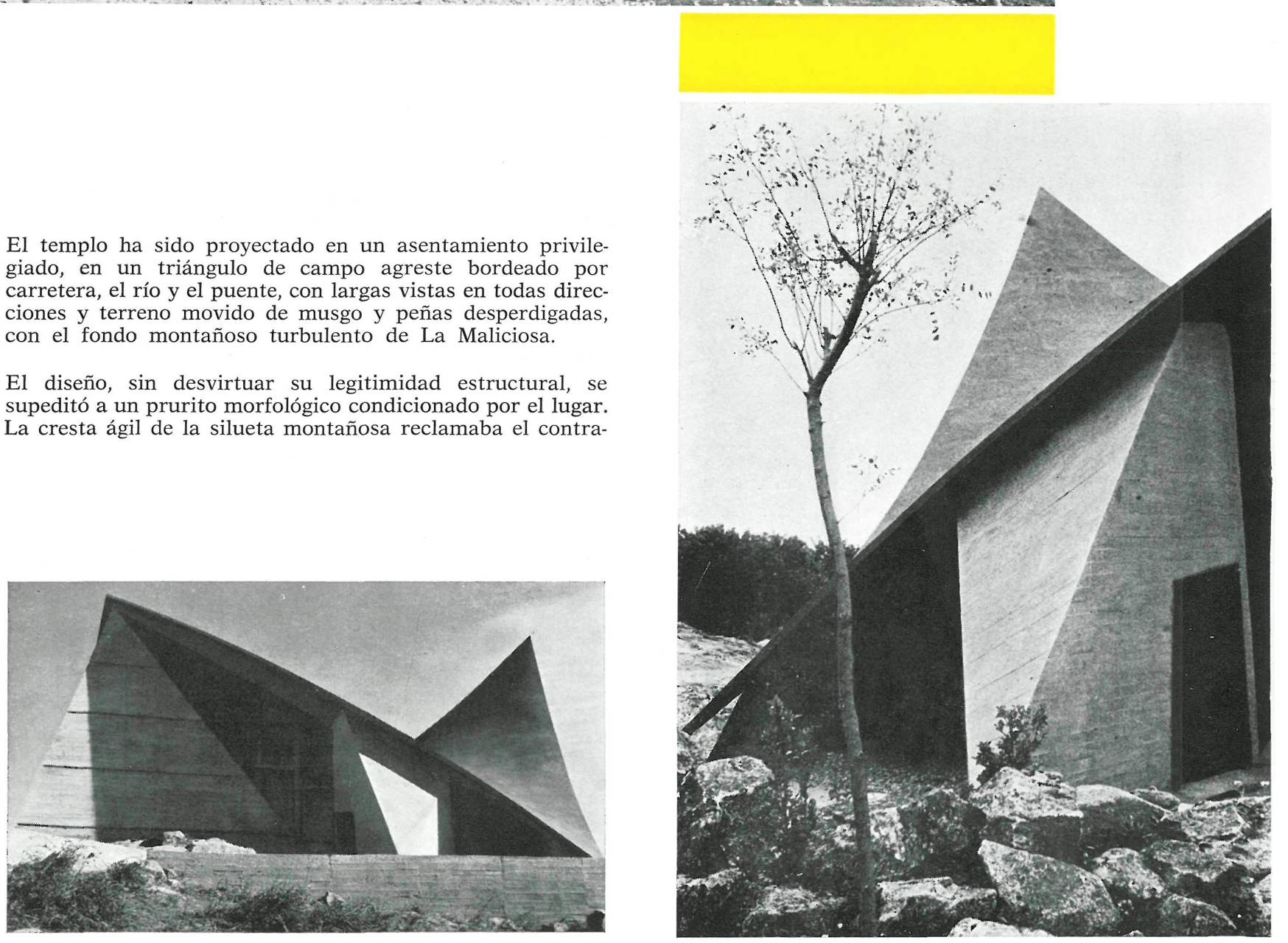
punto del perfil curvilíneo del triángulo de la cubierta. Tal configuración es poco usual en bóvedas laminares de estas dimensiones, por la imposibilidad de liberar de esfuerzos normales dos bordes contiguos. Pero estableciendo un contorno con bordes rectos, el diseño perdía la mayor parte de la gracia animal que ahora posee. En efecto, la bóveda en este caso tenía que emular al pájaro o a la hoja; había que buscar soluciones formales sin la rotundidad del paralelogramo, en cierto sentido ingrávidas, capaces de posarse sobre el espacio interior con delicadeza.

Por fin se seleccionó para la cubierta un paraboloide hiperbólico de curvatura más bien escasa e inclinado hacia delante un ángulo de $7^{\circ} 30^{\prime}$. En virtud de esta inclinación no se eleva desmesuradamente el vértice sobre el altar, y los cortes por planos verticales, de sencillo replanteo, producen en el escorzo curvas suficientemente expresivas.

Los bordes curvos, cargados con esfuerzos tangenciales y normales, era necesario sujetarlos desde abajo, ya que su excesiva longitud no permitía robustecerlos por medio de vigas, cuyo peso propio hubiera sido prohibitivo. La sustentación, pues, adquiere gran importancia desde el punto de vista de la composición, tanto o más que la cubierta.

La estructura no se sostiene de una manera tajante y clara. Fue preferible dotarla de un amplio margen de defensas concediéndole cierta arbitrariedad en la manera de conducir los esfuerzos al terreno, aun con detrimento de la brillantez intelectual que pudiera exhibir otra solución más limpia. El análisis se basa en principios físicos más que matemáticos.

La inclinación de los muros laterales es muy interesante por la cantidad de espacio ganado en planta, y mucho más, desde el punto de vista volumétrico exterior. Además la lámina es muy presumible que esté empujándolos hacia fuera, acción que contrarresta en parte el vuelco de viento más peso propio dirigido hacia dentro, contrarrestado además por las aletas que despiezan la pared exterior.

El espesor de la lámina es de $4 \mathrm{~cm}$. Dicho espesor aumenta hasta $10 \mathrm{~cm}$ en toda una visera trasera fuerte, estabilizada por los macizos bastante escultóricos de la entrada, y hasta $18 \mathrm{~cm}$, en el refuerzo existente sobre el altar. Estas diferencias quedan embebidas y disimuladas por el espesor del hormigón celular que posteriormente se extendió por encima.

La gran cantidad de masa de hormigón aparente hubiera resultado cansada, y es por eso por lo que se ha pretendido establecer una diferenciación, en parte por un enérgico despiece horizontal sobre las pirámides coincidiendo con las juntas del hormigonado, y por otro lado lavando la superficie de los muros inclina-

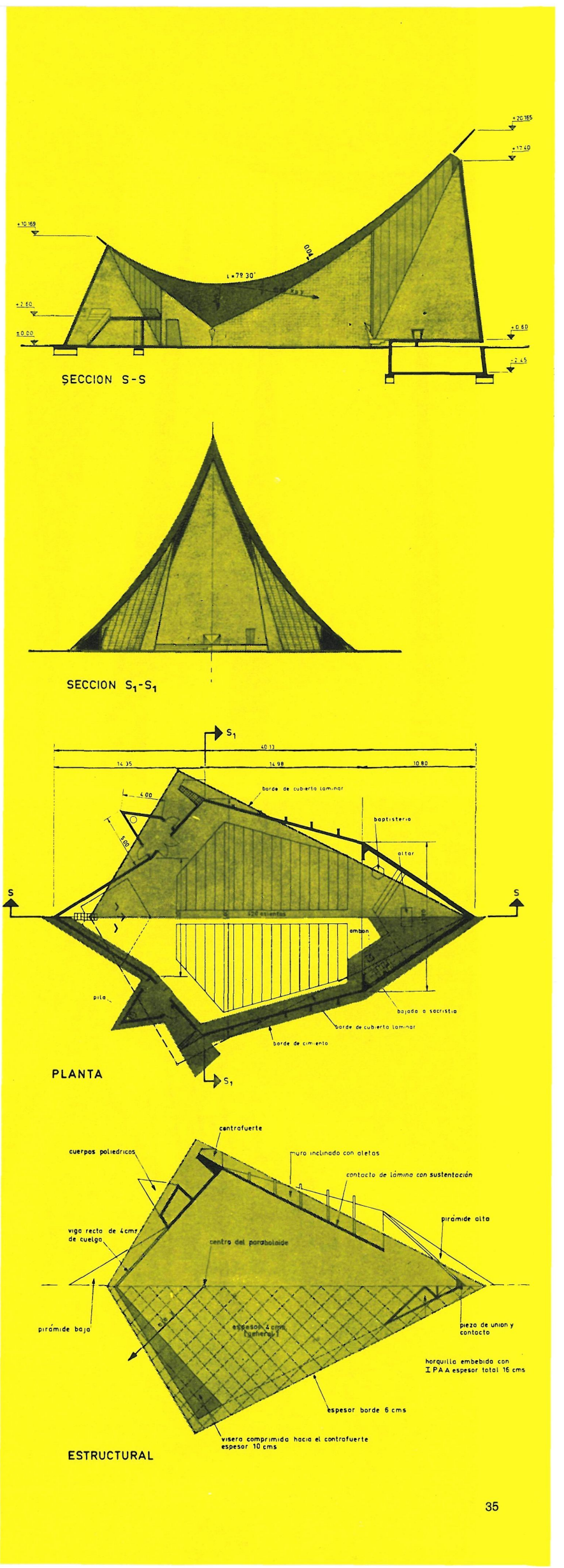



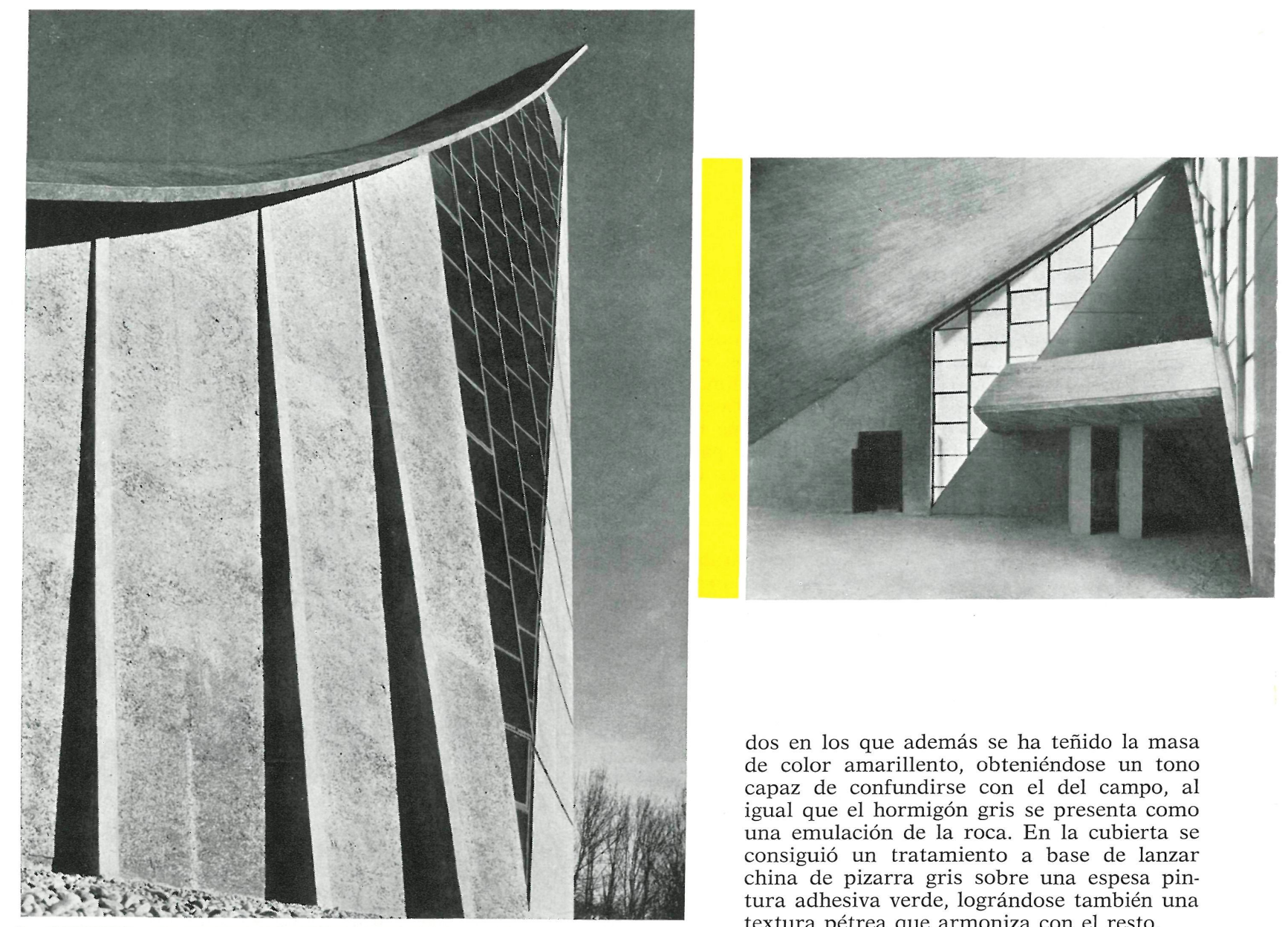

dos en los que además se ha teñido la masa de color amarillento, obteniéndose un tono capaz de confundirse con el del campo, al igual que el hormigón gris se presenta como una emulación de la roca. En la cubierta se consiguió un tratamiento a base de lanzar china de pizarra gris sobre una espesa pintura adhesiva verde, lográndose también una textura pétrea que armoniza con el resto.

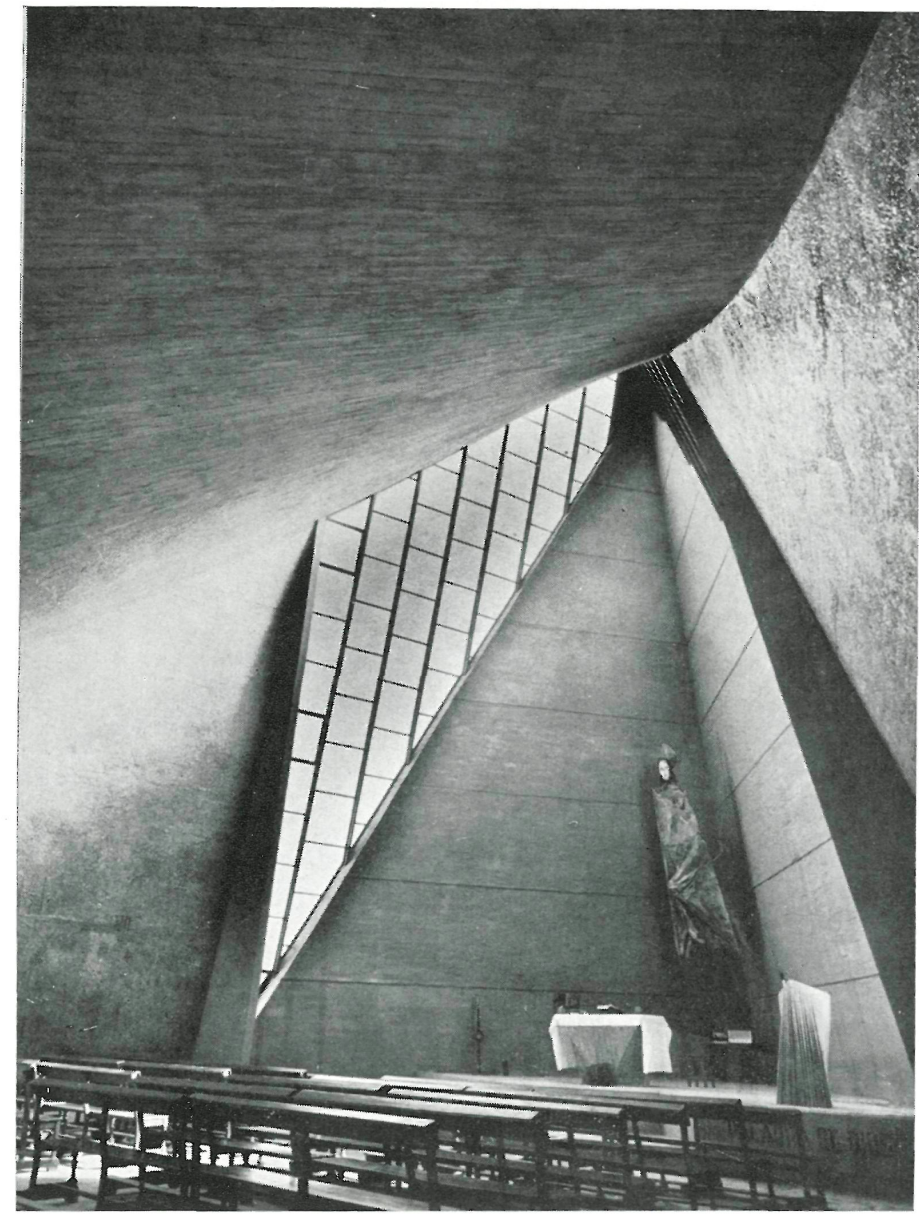

Parece haberse conseguido que el edificio se defina con el mimetismo del camaleón, integrándose bastante biológicamente al campo y resultando raramente cambiante con las horas y las estaciones.

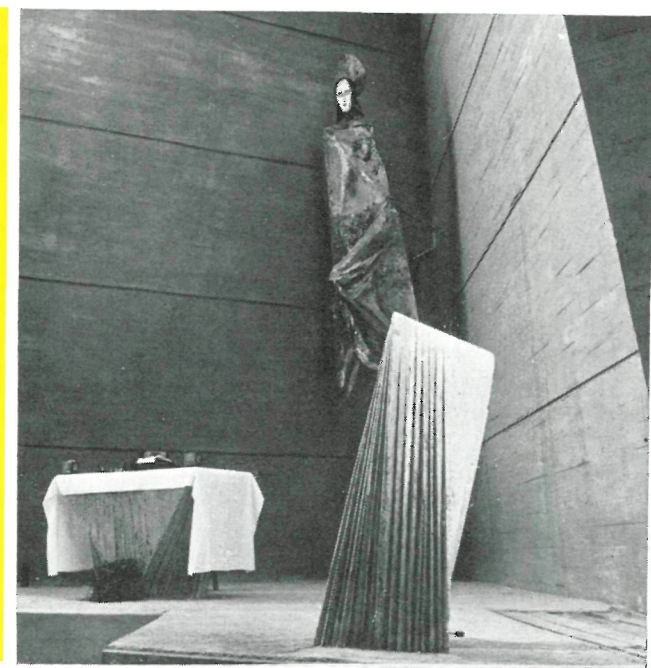

FOOS. BENUAMIN GUTIERREZ 


\section{Eglise ì Becermil de la Siemm - Espogne}

Architectes: Fray Coello de Portugal, R. Urgoiti et J. Ruiz del Castillo, Construcciones Laminares, S. L.

Toute la grâce de cette église réside dans l'heureux choix formel de son plan et dans l'absence de pesanteur de son voile mince - qui est un paraboloïde hyperbolique de faible courbure ayant une épaisseur minimale d $\mathrm{d} 4 \mathrm{~cm}$ et une épaisseur maximale de 10 et $18 \mathrm{~cm}$ - qui l'assimile à un oiseau ou à une feuille.

L'emplacement privilégié et le grand mimétisme obtenu avec le paysage environnant augmentent le charme naturel et spontané de ce temple.

\section{Ghumeh; at Becempil de Ia Siempa - Spain}

Architects: Fray Coello de Portugal, R. Urgoiti \& J. Ruiz del Castillo, Construcciones Laminares, S. L.

The main charm of this church depends on the right choice of the planform shape and of the extreme lightness of its thin shell roof, which is a hyperbolic paraboloid of slight curvature; its minimum thickness being $4 \mathrm{~cm}$ and its maximum thickness varies between 10 and $18 \mathrm{~cm}$. Its general shape is reminis. cent of a bird wing of smooth contours.

The outstanding site and its beautiful colour harmony with the surrounding landscape increase the natural and spontaneous charm of this building.

\section{Kirche in Becermil de Ia Sierna - Spanien}

Architekten: Fray Coello de Portugal, R. Urgoiti und J. Ruiz del Castillo, Construcciones Laminares, S. L.

Die grosse Anmut der Kirche beruht auf der Gestaltung des Grundrisses und der Schwerelosigkeit der Decke - ein leicht gekrümmtes hyperbolisches Paraboloid mit einer Dicke von $4 \mathrm{~cm}$ als Minimum und 10 und $18 \mathrm{~cm}$ als Maximum - die an einen Vogel oder ein Blatt mit zarten Umrissen erinnert.

Die bevorzugte Lage und die grosse Anpassung an die Umgebung steigern die grosse Anmut des Gebäudes. 